\title{
Lack of Kcnn4 improves mucociliary clearance in muco-obstructive lung disease
}

\author{
Génesis Vega, ${ }^{1}$ Anita Guequén, ${ }^{1,2}$ Amber R. Philp, ${ }^{1,2}$ Ambra Gianotti, ${ }^{3}$ Llilian Arzola, ${ }^{4}$ \\ Manuel Villalón, ${ }^{4}$ Olga Zegarra-Moran, ${ }^{3}$ Luis J.V. Galietta, ${ }^{5,6}$ Marcus A. Mall,, ${ }^{7,8}$ and Carlos A. Flores ${ }^{1}$ \\ ${ }^{1}$ Centro de Estudios Científicos, Valdivia, Chile. ${ }^{2}$ Universidad Austral de Chile, Valdivia, Chile. ${ }^{3}$ Istituto Giannina Gaslini, \\ Genoa, Italy. ${ }^{4}$ Departamento de Fisiología, Pontificia Universidad Católica de Chile, Santiago, Chile. ${ }^{5}$ Telethon Institute \\ of Genetics and Medicine, Pozzuoli, Italy. ${ }^{6}$ Department of Translational Medical Sciences, University of Naples \\ Federico II, Naples, Italy. 'Department of Pediatric Pulmonology, Immunology and Critical Care Medicine, Charité - \\ Universitätsmedizin Berlin, Berlin, Germany. ${ }^{8}$ Berlin Institute of Health, Berlin, Germany. ${ }^{9}$ German Center for Lung \\ Research, Berlin, Germany.
}

Airway mucociliary clearance (MCC) is the main mechanism of lung defense keeping airways free of infection and mucus obstruction. Airway surface liquid volume, ciliary beating, and mucus are central for proper MCC and critically regulated by sodium absorption and anion secretion. Impaired MCC is a key feature of muco-obstructive diseases. The calcium-activated potassium channel KCa.3.1, encoded by Kcnn4, participates in ion secretion, and studies showed that its activation increases $\mathrm{Na}^{+}$absorption in airway epithelia, suggesting that КСа3.1-induced hyperpolarization was sufficient to drive $\mathrm{Na}^{+}$absorption. However, its role in airway epithelium is not fully understood. We aimed to elucidate the role of KCa3.1 in MCC using a genetically engineered mouse. КСa3.1 inhibition reduced $\mathrm{Na}^{+}$absorption in mouse and human airway epithelium. Furthermore, the genetic deletion of Kcnn4 enhanced cilia beating frequency and MCC ex vivo and in vivo. Kcnn4 silencing in the Scnn1b-transgenic mouse (Scnn1 $b^{\text {tg/ }+}$ ), a model of muco-obstructive lung disease triggered by increased epithelial $\mathrm{Na}^{+}$absorption, improved MCC, reduced $\mathrm{Na}^{+}$absorption, and did not change the amount of mucus but did reduce mucus adhesion, neutrophil infiltration, and emphysema. Our data support that KCa3.1 inhibition attenuated muco-obstructive disease in the Scnn1 $b^{\text {tg/+ }}$ mice. $\mathrm{K}^{+}$ channel modulation may be a therapeutic strategy to treat muco-obstructive lung diseases.

Conflict of interest: The authors have declared that no conflict of interest exists

Submitted: May 8, 2020

Accepted: July 8, 2020

Published: August 20, 2020

Reference information: /CI Insight. 2020;5(16):e140076.

https://doi.org/10.1172/jici. insight.140076.

Copyright: $\odot$ 2020, Vega et al. This is an open access article published under the terms of the Creative Commons Attribution 4.0 International License.

\section{Introduction}

Mucociliary clearance (MCC) is a key process, which sustains airway innate defense, and its proper function relies on coordinate regulation of epithelial ion and fluid transport, mucus homeostasis, and ciliary beating $(1,2)$. Disruption of any of these epithelial functions reduces airway clearance capacity, as observed in asthma, chronic obstructive pulmonary disease (COPD), idiopathic pulmonary fibrosis or primary ciliary dyskinesia, all lung diseases characterized by mucus accumulation, airway obstruction, infections, and progressive bronchiectasis (3-5). Cystic fibrosis (CF), the most common genetic disorder in humans, is the "flagship" of all muco-obstructive diseases. Mutations in the CFTR gene impede anion transport through the CFTR protein, which reduce lung function dramatically (6). The absence of chloride secretion into the airway lumen dehydrates the airway surface liquid (ASL) and impairs cilia movement, whereas absent $\mathrm{HCO}_{3}^{-}$secretion (that can be secondary to $\mathrm{Cl}^{-} / \mathrm{HCO}_{3}^{-}$exchange or directly through CFTR) affects mucins deployment, making it sticky and hard to transport. Reduced $\mathrm{HCO}_{3}^{-}$secretion also produces acidic $\mathrm{pH}$, which in turn decreases activity of ASL antimicrobial molecules, favoring infection settlement (7).

Although there is an ongoing discussion regarding the existence of increased $\mathrm{Na}^{+}$absorption in $\mathrm{CF}$ tissues (8-11), its contribution to airway malfunction is tangible in a transgenic animal model with epithelial $\mathrm{Na}^{+}$channel $(\mathrm{ENaC})$ hyperactivity in the airways, which induce a muco-obstructive and inflammatory lung disease phenotype that shares key features with CF and COPD $(12,13)$. This observation goes in hand with the attempts to use ENaC channel blockers as a therapy for patients with $\mathrm{CF}$ that have shown improvements in mucus clearance but of short duration (14-16) or with occurrence of renal side effects (17). Genetic silencing using siRNA-based strategies directed against $\mathrm{ENaC}$ produce long-lasting increase in ASL and cilia beating frequency of human bronchial epithelial cells (HBECs) from patients with CF $(18,19)$, indicating again that reduction of $\mathrm{Na}^{+}$absorption is of potential benefit in $\mathrm{CF}$ and hence other muco-obstructive disorders. 
The mechanism for $\mathrm{Na}^{+}$absorption, as described by Koefoed-Johnsen and Ussing in the late 1950s, requires basolateral $\mathrm{K}^{+}$channel activity to sustain the electrical membrane gradient (20), and so $\mathrm{Na}^{+}$ absorption in the airways can be hypothetically regulated by basolateral $\mathrm{K}^{+}$channel function. Tens of $\mathrm{K}^{+}$ channels are expressed in the airway epithelium, but in most cases their role in ion transport mechanisms of the lower airway epithelium has not been tested (21).

In this study, we determined the role of the KCa3.1 channel using inhibitors and Kcnn4 silencing in ion transport and MCC. Using an animal model of CF/COPD-like muco-obstructive lung disease, we generated a double mutant animal to test the hypothesis that $\mathrm{Kcnn} 4$ silencing ameliorates airway disease.

\section{Results}

$\mathrm{Na}^{+}$absorption and calcium-activated anion secretion is dependent on KCa3.1 activity in mouse and human airway epithelium. First, we aimed to determine if the genetic deletion of Kcnn4 affected electrogenic ion transport in the airway epithelium of mouse trachea. Representative traces of freshly excised WT and $K c n n 4^{-1-}$ tracheal tissues are presented in Figure 1, A and B. We observed that the transepithelial potential $\left(\mathrm{V}_{\mathrm{te}}\right)$ was significantly reduced in the $\mathrm{Kcnn}^{\mathrm{S}^{-}}$trachea when compared with WT trachea (Figure 1C). We observed no changes in transepithelial resistance $\left(\mathrm{R}_{\mathrm{te}}\right)$ (Figure 1D) or basal short-circuit current $\left(\mathrm{I}_{\mathrm{sc}}\right)$ (Figure 1E). The amiloride-sensitive current was significantly reduced in the $K c n n 4^{-1}$ tissues (Figure $1 \mathrm{~F}$ ). Both the amiloride-insensitive (Figure $1 \mathrm{G}$ ) and the cAMP-induced anion secretion (Figure $1 \mathrm{H}$ ) remained unaffected by Kcnn4 silencing. The peak response of $\mathrm{Ca}^{2+}$-activated anion secretion induced by uridine-5'-triphosphate (UTP) (Figure 1I) and the plateau phase ( 5 minutes after UTP addition; Figure $1 \mathrm{~J}$ ) were reduced. Bioelectrical parameters are summarized in Table 1. We also tested if the silencing of another $\mathrm{K}^{+}$channel, the KCNQ1/KCNE3, that is also important for anion secretion affected $\mathrm{Na}^{+}$absorption using $\mathrm{Kcne}^{-/-}$tracheae, but we observed no changes (Table 1).

To determine if KCa3.1 inhibition mimics the observations in mouse trachea, we next tested known inhibitors of KCa3.1 channels, clotrimazol and TRAM-34, in HBEC monolayers mounted in Ussing chambers. Clotrimazol $(30 \mu \mathrm{M})$ did not affect amiloride-sensitive $\mathrm{Na}^{+}$absorption but produced a significant reduction in both cAMP- and $\mathrm{Ca}^{2+}$-activated anion secretion (Supplemental Figure 1, A-C; supplemental material available online with this article; https://doi.org/10.1172/jci.insight.140076DS1). TRAM-34 produced a significant reduction of the amiloride-sensitive current when used at $300 \mathrm{nM}$ (Figure $1, \mathrm{~K}$ and L), with no effect on CAMP- or $\mathrm{Ca}^{2+}$-activated anion secretion (Supplemental Figure 1, B and C). When TRAM-34 was used at $100 \mathrm{nM}$, there was no effect on any of the measured parameters (Supplemental Figure 1, A-C).

To dispense with the idea that reduction in the amiloride-sensitive current is produced by a reduced expression of $\mathrm{ENaC}$ subunits or by a reduction in the epithelial protease and $\mathrm{ENaC}$ activator prostasin (Prss8), we performed qRT-PCR analysis and demonstrated that all ENaC subunits and Prss 8 gene expression remained unaltered after Kcnn4 silencing (Supplemental Figure 2).

KCa3.1 inhibition increases ciliary beating frequency and clearance in mouse airways. To test if reduced $\mathrm{Na}^{+}$ absorption affected MCC, we studied changes in UTP-induced ciliary beating frequency (CBF), as determined in $\mathrm{Kcnn}^{\mathrm{H}^{--}}$or WT mouse airway cells cultures incubated with TRAM-34 or/and amiloride. As observed in Figure 2A, genetic silencing or TRAM-34 increased UTP-induced CBF. The summary in Figure $2 \mathrm{~B}$ includes experiments with amiloride with or without addition of TRAM-34. Amiloride alone did not significantly increase CBF, except when combined with TRAM-34.

Since CBF experiments must be performed in submerged conditions, we tested the clearance of plastic beads in freshly excised mouse tracheae, whose mucosal side was exposed to air in a humidified chamber. Figure 2, C and D, shows polar plots for WT and $\mathrm{Kcnn}^{-1-}$ tissues in which an increase in the distance covered by the beads is clearly seen. Detailed analysis of the movement of the beads showed that $K c n n 4$ silencing induced a significant increase in the average speed (Figure $2 \mathrm{G}$ ) and the total covered distance (Figure 2H). To evaluate if inhibition of $\mathrm{Na}^{+}$absorption affected the speed and distance traveled by the plastic beads, we used amiloride (Figure 2E) and observed an increase in both parameters in WT tracheae (Figure 2, G and H), as previously demonstrated (22). Nevertheless, when amiloride was tested in the $\mathrm{Kcnn}^{-1-}$ tracheae, a decrease in MCC parameters was observed compared with nontreated $\mathrm{Kcnn4^{-1 }}$ tracheae (Figure 2F), but still maintained higher MCC parameters than control WT tissues (Figure 2, G and H). Finally, we tested whole lung clearance in vivo, measuring the clearance of fluorescent-labeled OVA and found a higher clearance percentage in the $\mathrm{Kcnn}^{-{ }^{--}}$animals correlating with the ex vivo observations and demonstrating that Kcnn4 silencing increases MCC (Figure 2I).

Genetic silencing of Kcnn4 reduces $\mathrm{Na}^{+}$absorption and increases MCC in the Scnn $1 b^{\text {tg/ }}{ }^{+}$mouse. To test if the improved MCC observed in the $K c n n 4^{-1}$ mouse might be of potential benefit, we mated the $K c n n 4^{--}$animals with the 
A

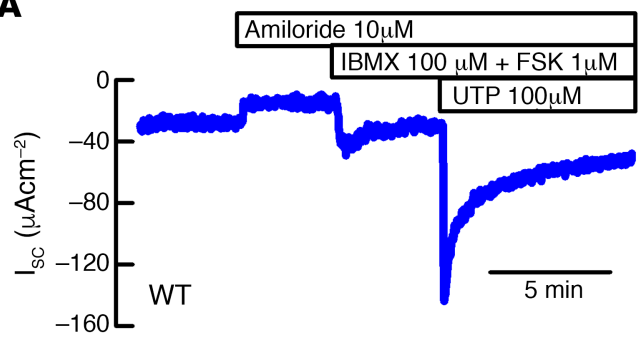

C

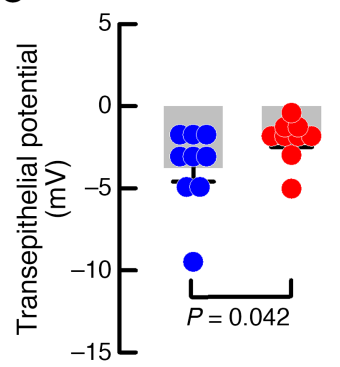

G

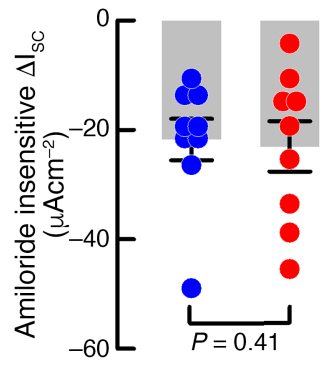

D

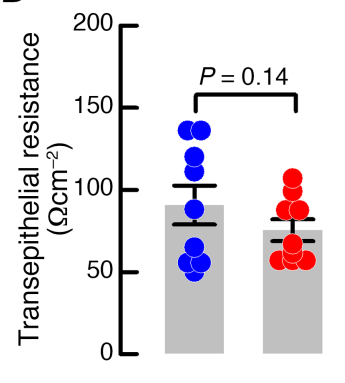

H

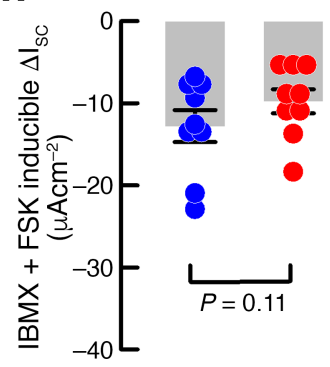

B

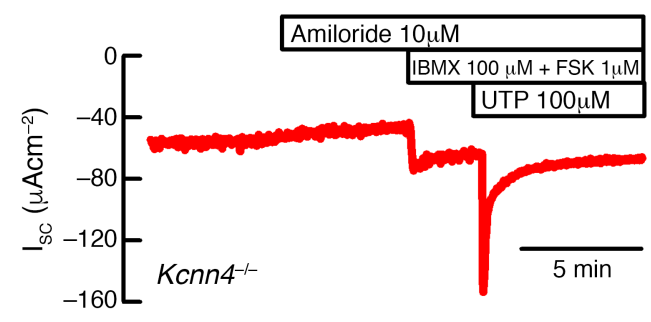

E

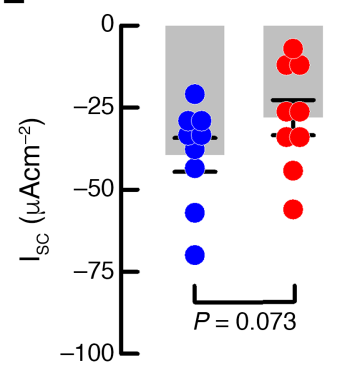

I

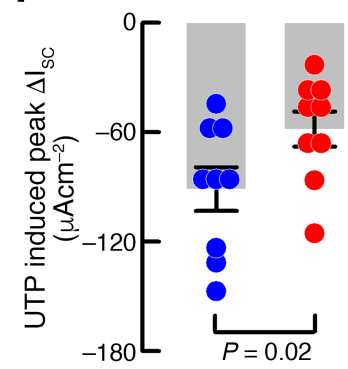

$\mathbf{F}$

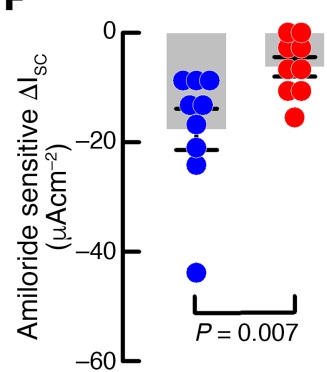

K

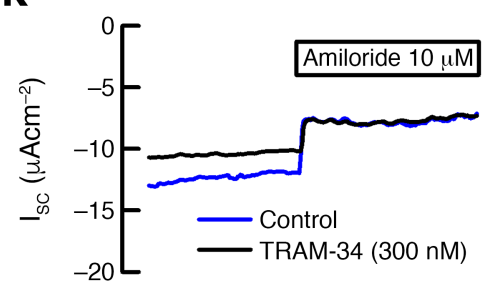

$\mathbf{L}$

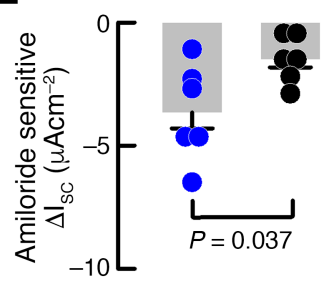

J

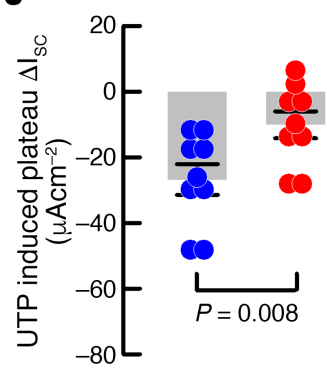

Figure 1. KСа3.1 participates in sodium absorption and anion secretion of mouse and human epithelium. Representative short-circuit current traces of

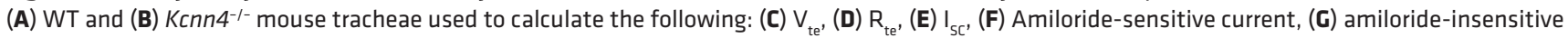
current, (H) cAMP-induced anion current and $\mathrm{Ca}^{+2}$-activated anion current at (I) peak or (J) plateau phases; $n=9$ for each group. (K) Representative Ussing chamber recordings of HBECs incubated with TRAM-34 and controls showing amiloride addition. (L) Summary of TRAM-34 effect on amiloride-sensitive $\mathrm{Na}^{+}$absorption; $n=6$ different cell cultures for each condition. Statistical differences were calculated using rank-sum test. Detailed values including data for $\mathrm{Kcne}^{-1-}$ are included in Table 1.

Scnn $1 b^{\mathrm{tg} /+}$ mouse that is affected by severe inflammatory and muco-obstructive airway disease. First, we studied if Konn4 silencing induced changes in epithelial $\mathrm{Na}^{+}$absorption and airway clearance. Ussing chamber experiments demonstrated that amiloride-sensitive $\mathrm{Na}^{+}$absorption was significantly decreased in double mutants compared with tracheae from the Scnn $1 b^{\text {tg/+ }}$ mouse (Figure 3, A and B), reaching values similar to those observed in the WTs (Table 1). The clearance of beads also showed increased values for speed and distances (Figure 3, C-E), indicating that Kcnn4 silencing improved MCC in the muco-obstructive lung disease model. 
Table 1. Bioelectrical parameters of mouse tracheae

\begin{tabular}{|c|c|c|c|c|c|}
\hline$V_{\text {te }}(m V)$ & $-3.76 \pm 0.8$ & $-2.04 \pm 0.4^{A}$ & $-1.07 \pm 0.3^{A}$ & $-3.51 \pm 0.6$ & $-2.24 \pm 0.4$ \\
\hline $\mathrm{R}_{\mathrm{te}}\left(\Omega / \mathrm{cm}^{2}\right)$ & $91 \pm 12$ & $75 \pm 6$ & $64 \pm 7$ & $59 \pm 7$ & $53 \pm 2$ \\
\hline $\mathrm{I}_{\mathrm{sc}}\left(\mu \mathrm{A} / \mathrm{cm}^{2}\right)$ & $-39.4 \pm 5$ & $-28.1 \pm 5$ & $-14 \pm 4^{A}$ & $-65 \pm 3^{A}$ & $-53.1 \pm 9$ \\
\hline Amiloride-sensitive $\left(\mu \mathrm{A} / \mathrm{cm}^{2}\right)$ & $-17.6 \pm 3.8$ & $-6.2 \pm 1.8^{A}$ & $-11.5 \pm 1.4$ & $-25.2 \pm 1.7^{A}$ & $-12.7 \pm 3^{B}$ \\
\hline IBMX+FSK-induced $\left(\mu \mathrm{A} / \mathrm{cm}^{2}\right)$ & $-12.8 \pm 1.9$ & $-9.7 \pm 1.4$ & n.d. & n.d. & n.d. \\
\hline UTP-induced peak $\left(\mu \mathrm{A} / \mathrm{cm}^{2}\right)$ & $-91.1 \pm 12$ & $-58.2 \pm 9.6^{A}$ & n.d. & n.d. & n.d. \\
\hline UTP-induced plateau $\left(\mu \mathrm{A} / \mathrm{cm}^{2}\right)$ & $-26.8 \pm 4.7$ & $-10.1 \pm 4.1^{\mathrm{A}}$ & n.d. & n.d. & n.d. \\
\hline
\end{tabular}

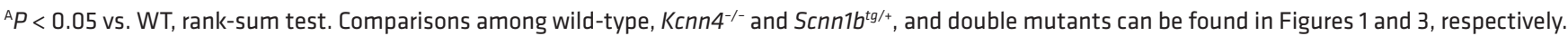

${ }^{B} P<0.05$ vs. Scnn1 $b^{\text {tg/+}}$, rank-sum test. FSK, forskolin; UTP, uridine-5'-triphosphate.

Genetic silencing of Kcnn4 prevents airway inflammation and lung damage in the Scnn1b ${ }^{\text {tg/+ }}$ mouse. After Kcnn4 silencing lung edema in the Scnn $1 b^{\text {tg/+ }}$ mouse was reduced to levels similar to the control animals (Figure 4A) and concomitantly, we observed less damage associated with emphysema (Figure 4, B and C). Analysis of bronchoalveolar lavage fluid (BALF) was performed to quantify the absolute numbers and relative distribution of immune cell types present in the airways of the animals. As previously observed (12), the Scn$n 1 b^{\mathrm{tg} /+}$ mouse showed increased amounts of total cells (Figure 4D), corresponding to macrophages (Figure $4 \mathrm{E}$ ) and neutrophils (Figure 4F). The silencing of Kcnn4 did not affect the number of total cells or macrophages but reduced the number of neutrophils in the $S c n n 1 b^{\mathrm{tg} /+}$ airways, an observation that was confirmed with staining and quantification of neutrophils in mucus plugs with LY6G/LY6C (Figure 4, G and H). The silencing of Kcnn4 alone did not produce changes in the measured parameters (Figure 4).

To test if the improvement in lung disease correlates with a decrease in mucostasis, we analyzed lung histological samples corresponding to proximal bronchi and distal bronchi stained with periodic acid-Schiff (PAS). Intraluminal and intraepithelial mucus volume were increased in the Scnn $1 b^{\text {tg/+ }}$ mouse compared with WTs and $\mathrm{Kcnn}^{-/}{ }^{-}$in both proximal and distal airways (Figure 5, A and B). After Kcnn4 silencing, values were not significantly different from those observed in the $S c n n 1 b^{\mathrm{tg} /+}$ mouse, but we observed a trend toward reduced intraluminal mucus obstruction in the distal airways of double mutants $K c n n 4^{-1-/ S c n n 1 b^{\mathrm{tg} /+} \text { mice }}$ that was not statistically significant based on the number of mice available for these studies (Figure 5A). A closer examination of the tissues led us to the observation that although mucus in the $S c n n 1 b^{\mathrm{tg} /+}$ mouse mostly adhered to the epithelial wall, this was not the case in the double mutant airways (Figure 5C). Quantification of the epithelial airway surface in contact with mucus demonstrated that there was a gradient of mucus adhesion to the surface of the epithelium (proximal to distal airway gradient) in the WT animals $(17.4 \% \pm$ $3 \%$ and $0.4 \pm 0.1 \%$ of epithelial surface attached to mucus; $P<0.001)$ that was maintained in the $K_{c n n 4^{-1}}$ tissues $(14.6 \% \pm 4.8 \%$ and $2.3 \% \pm 1.2 \% ; P=0.02$; Figure $5 \mathrm{D})$. Analysis of the $S c n n 1 b^{\mathrm{tg} /+}$ tissues showed a complete loss of the proximal to distal airway gradient, as both values were similar and higher in both proximal and distal airways $(45.0 \% \pm 6 \%$ and $45.1 \% \pm 9.1 \% ; P>0.4)$. Finally, a recovery of the proximal to distal airway gradient was observed after Kcnn4 silencing $(59.7 \% \pm 10.8 \%$ and $24.7 \% \pm 7.4 \% ; P=0.014)$.

\section{Discussion}

We identified $\mathrm{KCa} 3.1$ as a $\mathrm{K}^{+}$channel responsible for energizing the cell electrical gradient for $\mathrm{Na}^{+}$absorption in human and mouse airway epithelial cells. As predicted from previous observations, reduced $\mathrm{Na}^{+}$ absorption might enhance MCC function in the airways of the $K c n n 4^{-/}$mouse; therefore, we tested the potential of Kcnn4 silencing in the amelioration of airway muco-obstructive disease in the $S c n n 1 b^{\mathrm{tg} /+}$ mouse model. Kcnn4 silencing successfully decreased inflammatory lung damage, neutrophilic inflammation, and improved MCC in the Scnn $1 b^{\mathrm{tg} /+}$ animals. We found that Kcnn4 silencing also affected anion secretion; however, no signs of muco-obstructive disease were found in this model. 
A

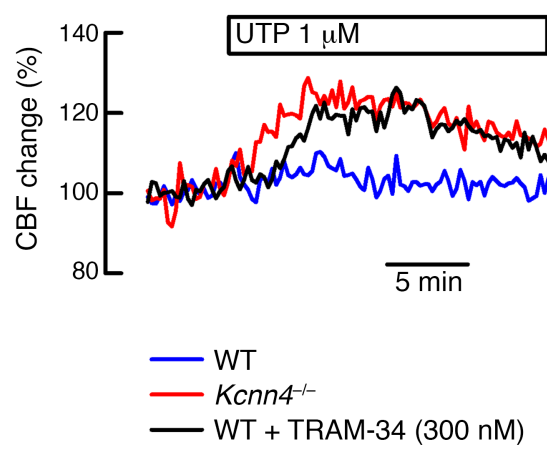

B

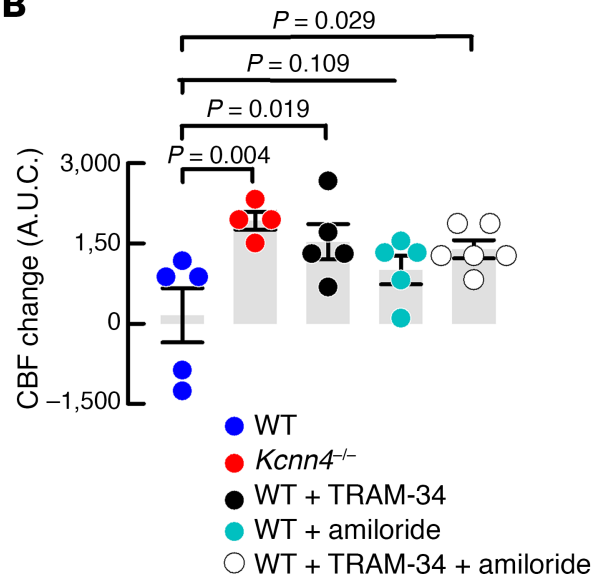

C

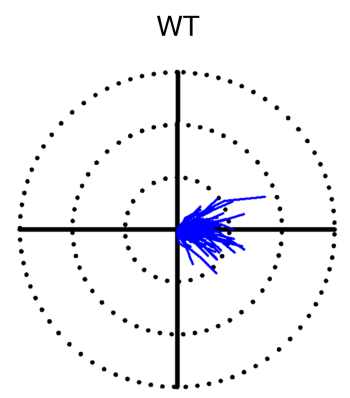

E

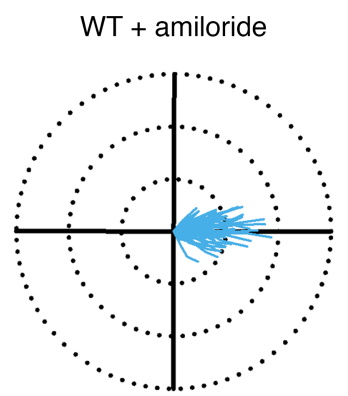

D

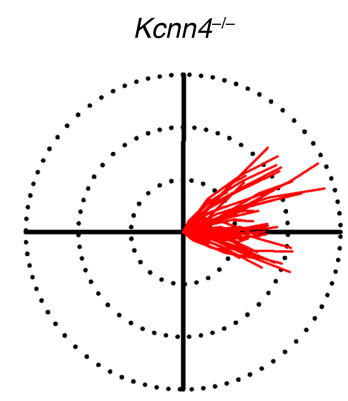

$\mathbf{F}$

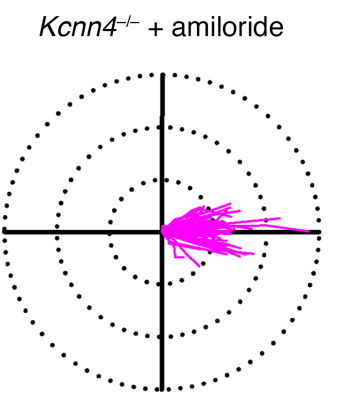

G

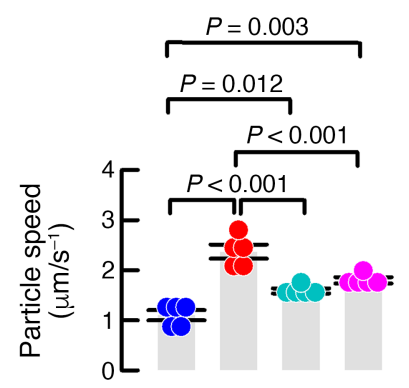

H

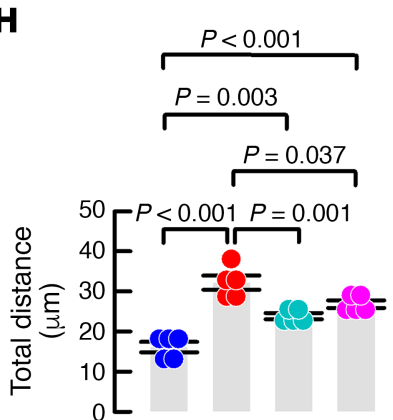

I

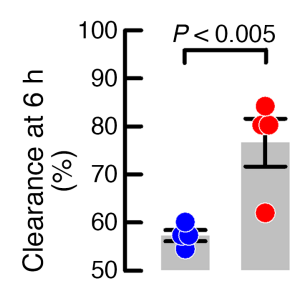

Figure 2. Mucociliary clearance is enhanced after KCa3.1 inhibition in the mouse. (A) CBF changes in Kcnn4-/- or WT cells treated with TRAM-34 (100 nM) after UTP stimulation of tracheal epithelial cell explants. (B) Quantification of the area under the curve of CBF recordings including WT explants treated with amiloride $(10 \mu \mathrm{M})$ and/or TRAM-34. Differences were calculated using ANOVA on ranks; $n=5,4,5,5$ and 6 , respectively. Polar plots (75 $\mu \mathrm{m}$ radius) of

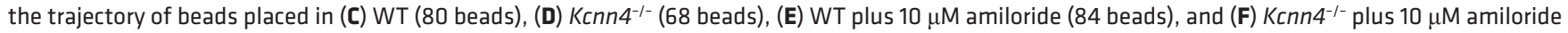
(81 beads) tracheae, corresponding to 5 different experiments for each group. Calculated speed of particles (G) and total distance (H) from the experiments shown in C-F. Differences were calculated using ANOVA on ranks. (I) In vivo lung clearance of fluorescently labeled OVA in WT $(n=4)$ and $K c n n 4^{-/-}(n=4)$ mice. Difference was calculated using rank-sum test. CBF, ciliary beating frequency; UTP, uridine-5'-triphosphate.

Early observations in patients affected with pseudohypoaldosteronism (due to ENaC-inactivating mutations) indicated that ENaC activity was inversely related to ASL volume and MCC efficiency (23), suggesting that $\mathrm{ENaC}$ might be a druggable target to manage muco-obstructive lung disease. Some rate of success is obtained using ENaC blockers, such as amiloride and derivatives, but short duration or side effects hampered further advances in the development of therapies and so new approaches are necessary (24). As described in the 1950 s, the $\mathrm{Na}^{+}$absorption mechanism relies on the activity of basolateral $\mathrm{K}^{+}$channels (20). Acting in concert with the $\mathrm{Na}^{+}$pump, highly selective basolateral channels allow the extrusion of $\mathrm{K}^{+}$that, if retained, depolarizes the membrane potential, hampering $\mathrm{Na}^{+}$absorption. This is the case in the kidney and intestine where inhibition 
A

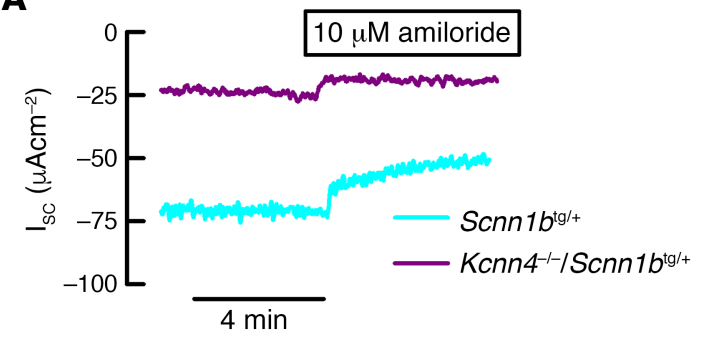

B

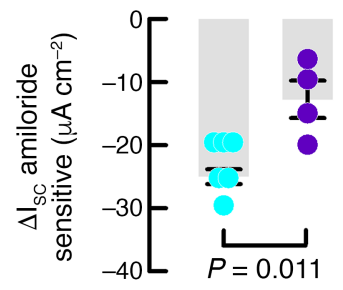

C

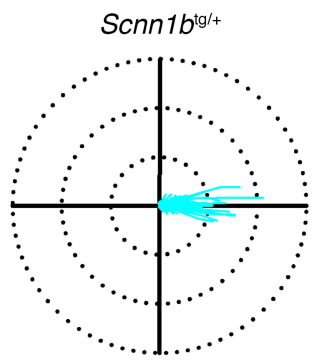

D

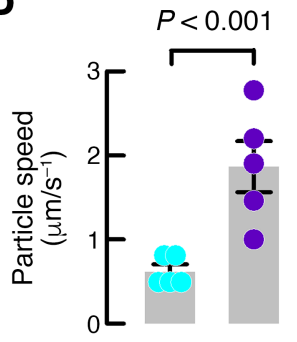

E
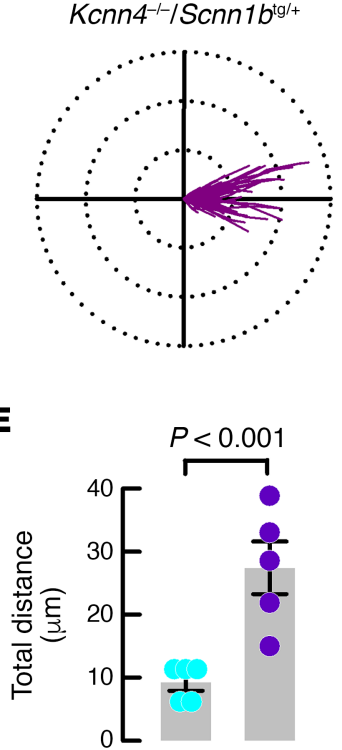

Figure 3. The genetic silencing of Kcnn4 reduces sodium absorption and increases MCC in the Scnn1 $b^{\text {tg//+ }}$ mouse trachea. (A) Representative $I_{s c}$ traces showing the extent of amiloride-sensitive currents in the $\operatorname{Scnn} 1 b^{\text {tg } /+}(n=6)$ and double mutant $(n=4)$ mice tracheae. (B) Summary of amiloride-sensitive currents as shown in $(\mathbf{A}), P$ value calculated using ANOVA on ranks. Polar plots ( $75 \mu \mathrm{m}$ radius) of the trajectory of beads placed in (C) Scnn1 $\mathrm{b}^{\text {tg/+ }}$ ( 222 beads) and double mutants (126 beads), from 5 different experiments each. Detailed electrical parameters are given in Table 1. Calculated (D) speed of particles and (E) total distance from the experiments shown in $\mathbf{C}$. Differences were calculated using ANOVA on ranks. MCC, mucociliary clearance.

of basolateral $\mathrm{K}^{+}$channels reduced $\mathrm{Na}^{+}$absorption and $\mathrm{Na}^{+}$-coupled metabolite uptake (25-27), further strengthening the role of $\mathrm{K}^{+}$channels on epithelial $\mathrm{Na}^{+}$homeostasis. We envisioned that $\mathrm{Na}^{+}$absorption in the airways can be controlled through basolateral $\mathrm{K}^{+}$channels, but their role in electrolyte transport has not been profusely studied in this tissue (21). With the exceptions of Kir7.1, KCNE3, and KCa3.1, no other $\mathrm{K}^{+}$ channels or subunits are located in the basolateral membrane of lower airway epithelium (28-30). Experiments with ion channel inhibitors demonstrate that KCNQ1/KCNE3, using chromanol-293B, and KCa3.1, using clotrimazole, participate in the mechanism of anion secretion of the airways, and experiments in the Kcne3-1mouse confirm its role, but no effect on $\mathrm{Na}^{+}$absorption is observed $(28,31,32)$. Our results confirmed these observations, as when using clotrimazole a decrease in anion secretion was observed, but there was no effect in $\mathrm{Na}^{+}$absorption in human cells, similar to what we observed in tracheae from the $\mathrm{Knne}^{3--}$ animals. When using the KCa3.1 opener 1-EBIO, we observe an increase in $\mathrm{Na}^{+}$absorption in HBECs, supporting a role for KCa3.1 as a regulator of $\mathrm{Na}^{+}$absorption in these cells (33), a result comparable with our observations using TRAM-34

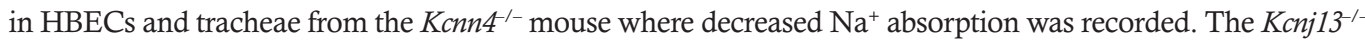
mouse bears a lethal phenotype at perinatal age and is affected by severe tracheomalacia, but it is not known if Kir7.1 participates in the anion secretion mechanism and if it relates to the observed phenotype (34).

Epithelial anion secretion in the airways is also affected after $\mathrm{K}^{+}$channel silencing that a priori, and in the scenario of a muco-obstructive disease, it is unwanted. Inhibition of both KCa3.1 and KCNQ1/KCNE3 produces a significant reduction of $\mathrm{Ca}^{2+}$ - and cAMP-induced anion secretion, respectively $(31,32)$, but no 
A

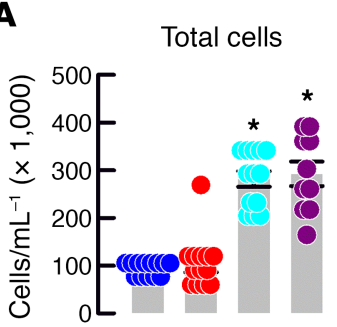

B
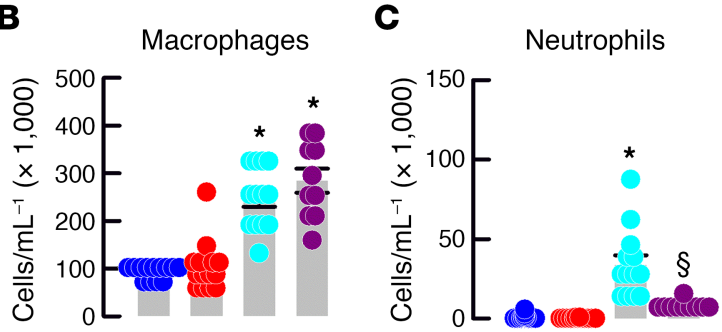

D

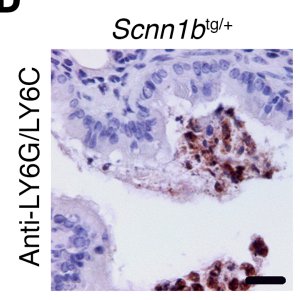

F

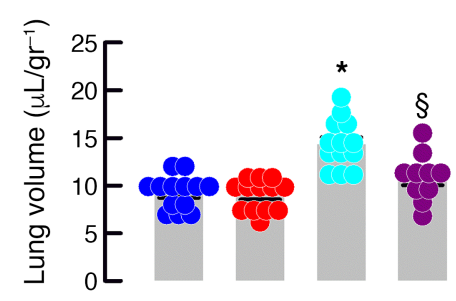

G

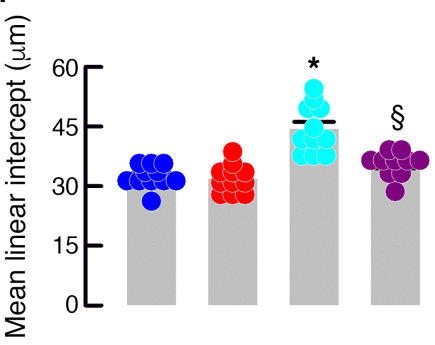

E
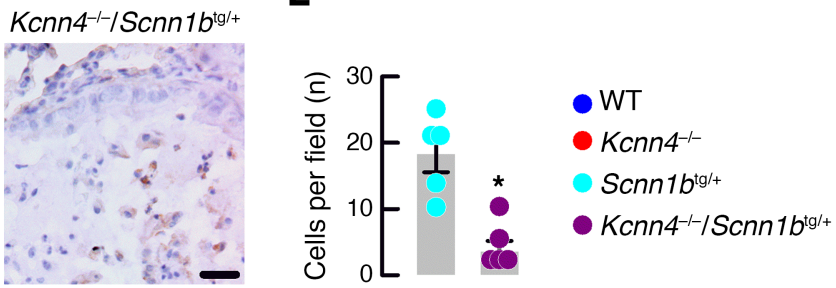

H
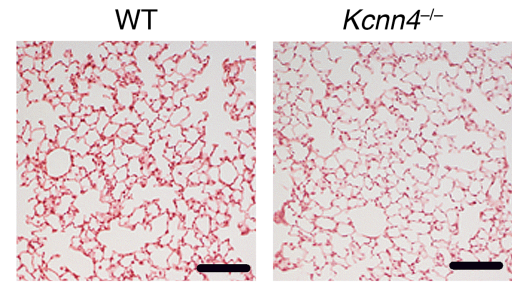

Scnn $1 b^{\mathrm{tg} / \mathrm{t}}$

Kenn4-1-/Scnn $1 b^{\text {tg//+ }}$
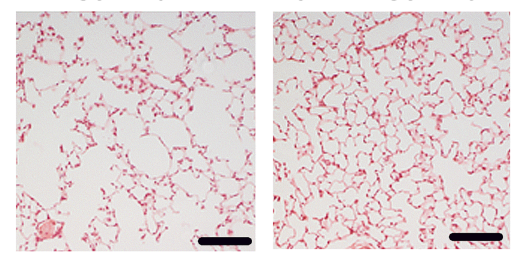

Figure 4. Genetic silencing of Kcnn4 reduces lung inflammatory disease in mice with muco-obstructive lung disease. Total cells (A) and macrophages (B) quantification in BALF. ${ }^{*} P<0.05$ vs. WT and $K c n n 4^{-1-}$. Neutrophils (C) quantification in BALF. ${ }^{*} P<0.05$ vs. all other groups and $\S$ indicates the difference vs. Scnn $1 b^{\text {tg } /+}$. ANOVA on ranks; $n=13,13,13$ and 10 for WT, $\mathrm{Knnn}^{+/+}, S \mathrm{cnn} 1 \mathrm{~b}^{\text {tg/+}}$, and double mutants, respectively. Representative images ( $n=5$ each group; scale bar: $20 \mu \mathrm{m}$ ) of LY6C/LY6C immunostaining in mucus plugs (D). Quantification of LY6C/LYGC-positive cells (E) in the Scnn $1 b^{\text {tg/+ }}(n=5)$ and double mutants $(n=5)$. ${ }^{*} P<0.05$ calculated by rank-sum test. Mouse lung volume $(\mathbf{F}) ;{ }^{*} P<0.05$ vs. all other groups and $\S$ indicates the difference vs. Scnn $1 b^{\text {tg/++}}$; ANOVA on ranks; $n=13,13,13$, and 10 for WT, Kcnn $4^{+/+}$, $S c n n 1 b^{t g / t}$, and double mutants, respectively. Mean linear intercept (C) calculated from images as shown in $\mathbf{H}$ (scale bar: $200 \mu \mathrm{m}) ;{ }^{*} P<0.05$ vs. all other groups and $\S$ indicates the difference vs. Scnn $1 b^{\mathrm{tg} /+}$; ANOVA on ranks; $n=13,13,13$, and 10 for WT, $\mathrm{Kcnn}^{+/+}$, Scnn1 $1 \mathrm{~b}^{\text {tg/+}}$, and double mutants, respectively.

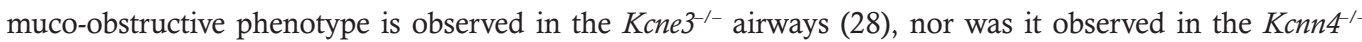
airways in our results. Such reductions in anion secretion are also innocuous in the intestine. For example, Kcnn4, Kcnq1, Kcne3, or Kcnk5 silencing does not produce obstructions or CF-like phenotypes in the intestine of the animals, as expected from genes coding for $\mathrm{K}^{+}$channels with tested roles in epithelial anion secretion $(26,28,35,36)$. Even though, we do not have a straight answer for the lack of the muco-obstructive phenotype when Kcnn 4 is silenced, it is important to consider that the inhibition of anion secretion in this model is not complete; KCNQ1/KCNE3 activity and basolateral electroneutral-anion influx can still support anion secretion at a sufficient level to maintain MCC function. 
A

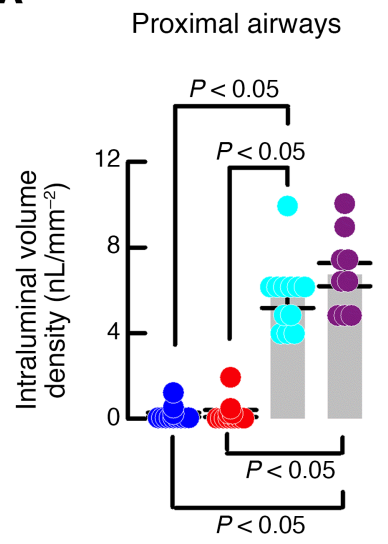

Distal airways

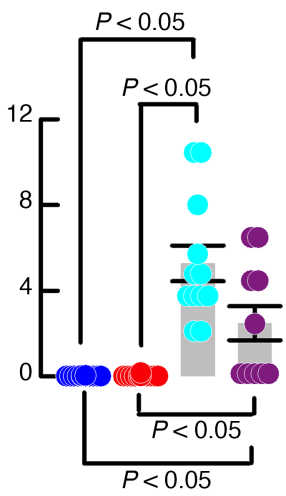

B
Proximal airways
Distal airways
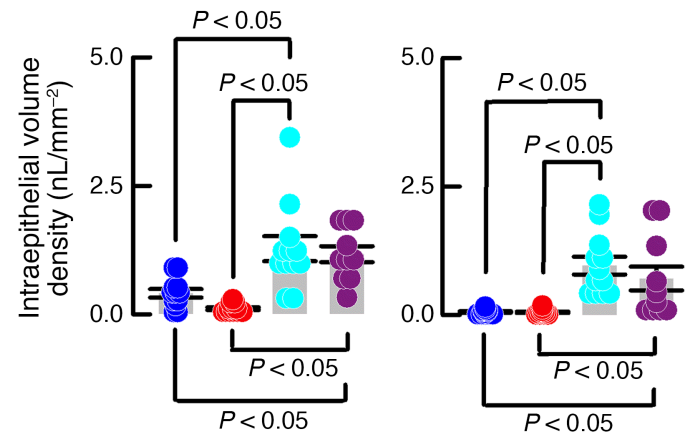

WT

Kcnn4-/-

Scnn $1 b^{\text {tg/t }}$

- Double mutant

C

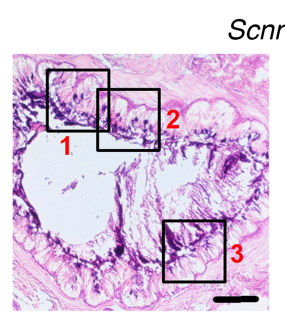

Scnn $1 b^{\mathrm{tg} /+}$
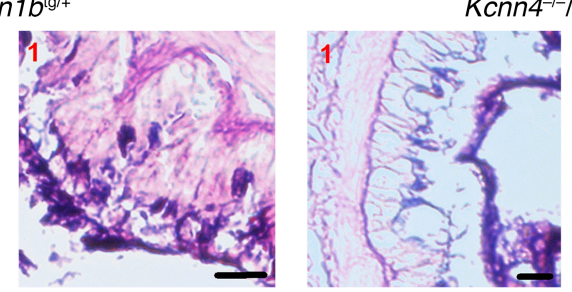

Kcnn $4^{-1 / S c n n} 1 b^{t g /+}$
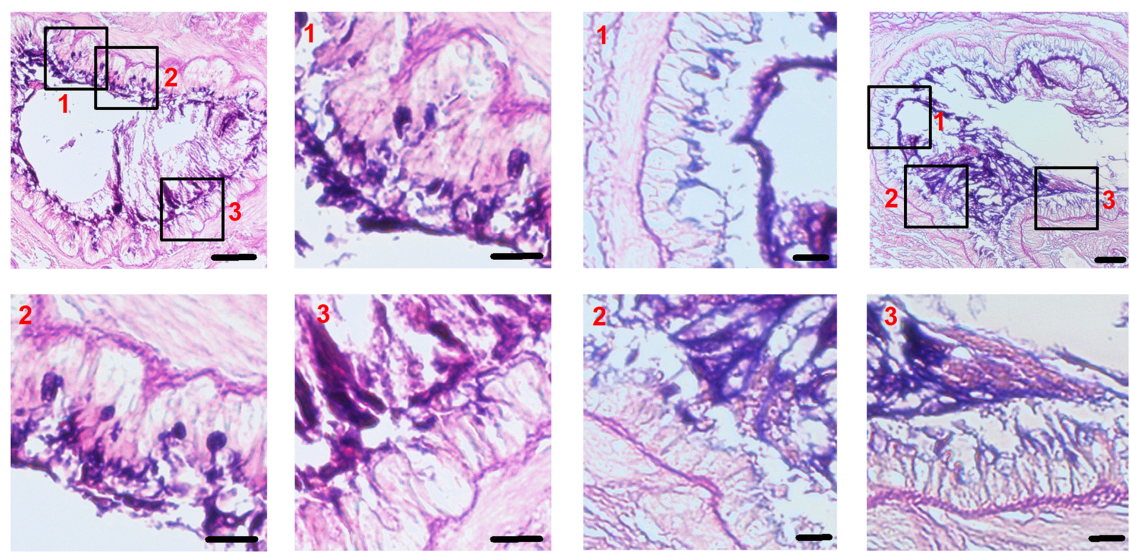

D

WT

$\mathrm{Kcnn}^{-1-}$

Scnn $1 b^{\text {tg/t }}$

Kcnn $4^{-1 / S c n n} 1 b^{t / /+}$
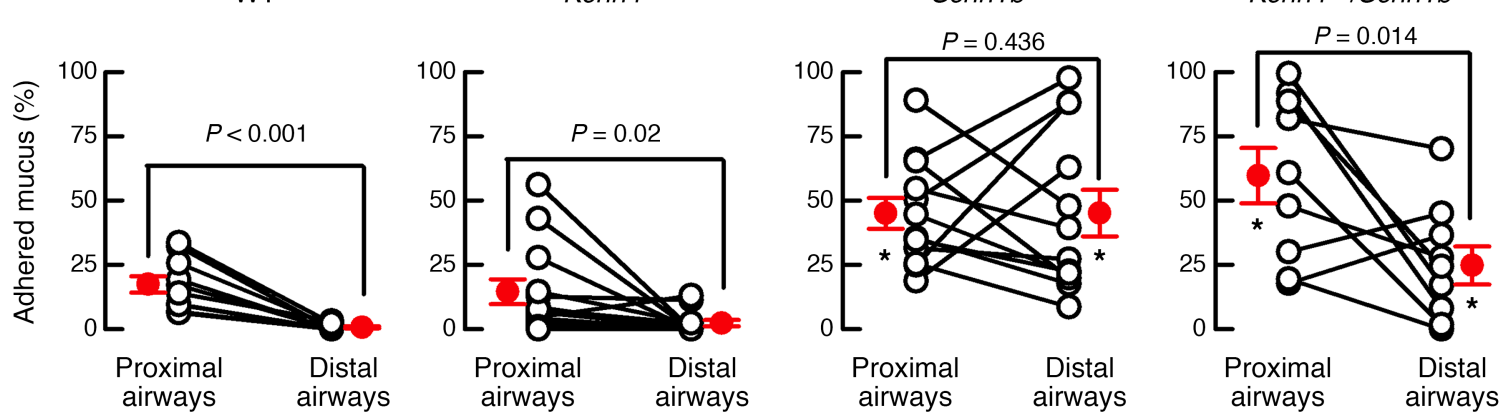

Figure 5. Genetic silencing of Kcnn4 improved mucus clearance in mouse airways. Intraluminal (A) and intracellular (B) mucus volume was determined in the proximal and distal airways. Differences were calculated with ANOVA on ranks; $n=12,12,12$ and 9 animals for WT, $K c n n 4^{+/+}$, Scnn1b ${ }^{\text {tg/+}}$, and double mutants, respectively. Representative images of mucus attachment to the epithelial surface for $\operatorname{Scnn} 1 b^{\text {tg } /+}(n=15)$ and double mutants $(n=13)(C)$.

Selected areas of main images (scale bar: $50 \mu \mathrm{m}$ ) are noted as 1-3 in red letters, and are shown amplified separately (scale bar: $20 \mu \mathrm{m}$ ). Summary of the percentage of epithelium surface covered my mucus in proximal and distal airways (D). Only paired samples from the same animal were included; $n=13$, 16,15 , and 13 for WT, $K c n n 4^{+/+}$, Scnn $1 b^{\text {tg/+/}}$, and double mutants, respectively. ${ }^{*} P<0.05$ vs. WT and $K c n n 4^{-/-}$ANOVA on ranks. The $P$ values for each proximal vs. distal airways comparison were calculated by rank-sum test.

But is inhibiting $\mathrm{KCa} 3.1$ better than inhibiting $\mathrm{ENaC}$ ? Besides the use of $\mathrm{ENaC}$ blockers and other inactivating strategies already discussed, animal models demonstrate that silencing of genes coding for $\mathrm{ENaC}$ subunits is lethal, and, in particular, Scnn1a silencing produces lethality due to defective liquid clearance from the neonatal lung $(37,38)$. Nevertheless, we agree with the idea that the key seems to be the dosage of ENaC inhibition, as the heterozygous mutant mice for Scnn1a do not show lethality, and residual ENaC activity in $\mathrm{Scnn}_{1 b^{-1}}$ and $\mathrm{Scnn} 1 \mathrm{~g}^{-1-}$ is sufficient to overcome the neonatal lung phenotype (38-40). Our results 
show that, after Kcnn4 silencing, approximately $35 \%$ of amiloride-sensitive $\mathrm{Na}^{+}$absorption was still active in mouse trachea, suggesting that the remaining current is sufficient to maintain proper MCC function.

Analysis of the genetic silencing of $K c n n 4$ in the $S c n n 1 b^{\text {tg/+ }}$ animal demonstrated reduced infiltration of neutrophils and decreased emphysemic damage of the respiratory epithelium. The reduction in neutrophils might be explained by a direct effect on the neutrophil's chemotaxis capacity that is reduced after Kcnn4 silencing (41), but additionally, the reduction of chemoattractants released might influence the arrival of inflammatory cells to the airways $(42,43)$. We also observed that a gradient of adhered mucus to the epithelium normally occurred, as mucus covers a larger surface in the proximal bronchi than in the terminal. This gradient is disrupted in the $S c n n 1 b^{\text {tg/t }}$ tissues, where similar values for mucus attached to the epithelial surface are observed in proximal and distal bronchi. Although we observed a tendency for mucus reduction in the distal airways of double mutants, it was accompanied by a recovery in the gradient of mucus adherence to the epithelial surface, supporting the idea that the mucus hypersecretory state per se is not enough to provoke muco-obstructive disease. For example, animals that overexpress MUC5AC or MUC5B in the lungs do not produce airway obstructions or inflammation, even when in some cases the amount of mucus protein is almost 20 times higher than that in control animals, thus reaffirming the idea that under normal fluid transport the amount of mucus is not an issue $(44,45)$. Mucus oversecretion must then be accompanied by airway dehydration and inflammatory signaling to complete the muco-obstructive pathology. Therefore, we conclude that the observed reduction of inflammation and the increased MCC after Kcnn4 silencing prevents the muco-obstructive pathology in the double mutant animals irrespective of mucus quantity. Even when mucus hypersecretion is at the center stage in airway muco-obstructive diseases, animal models demonstrate that impeding mucin expression is detrimental for animal well-being, because mucus is fundamental for lung health (45). Studying the same $S c n n 1 b^{\mathrm{tg} /+}$ mouse model with additional silencing of Muc5ac or Muc5b, increased inflammation or no improvements at all in airway disease is found (46). Our results agree with the idea that complete mucus reduction is not recommendable, but interventions that boost MCC, improve mucus quality, and/or reduce adhesion of mucus to the epithelium are in the road to limit the seriousness of the disease.

KCa3.1 possesses a high affinity for $\mathrm{Ca}^{2+}$, with $\mathrm{EC}_{50}$ values of 95-160 $\mathrm{nM}$ for the human and mouse channels, respectively $(47,48)$. Free calcium in the cytoplasm of airway epithelial cells is typically $100 \mathrm{nM}$ $(49,50)$, but ciliated cells bear a higher concentration at baseline than club cells (51). Another important functional consideration is that ciliated cells respond, increasing $\mathrm{Ca}^{2+}$ from $100 \mathrm{nM}$ up to $400 \mathrm{nM}$ when stimulated with a low ATP concentration $(0.1-1 \mu \mathrm{M})(52)$, close to what is normally found in the sputum and ASL from human donors (53-55). All this evidence argues in favor of the idea that KCa3.1 channels are active during normal conditions in certain cells of the airway epithelium, but further immunolocalization studies are necessary to unveil the exact cell distribution, subcellular localization, and protein interactions of KCa3.1 channels in the airways.

Even when decreased $\mathrm{Na}^{+}$absorption after $\mathrm{Kcnn} 4$ silencing fits with expected improvements in MCC, we have to address the fact that some of our results indicate that $\mathrm{KCa} 3.1$ functions go beyond epithelial fluid secretion and absorption. Increased $\mathrm{CBF}$ after $\mathrm{KCa} 3.1$ blockade occurs in submerged conditions, discarding a direct link to increased ASL. The hyperpolarization produced by the opening of $\mathrm{K}_{\text {ATP }}$ channels in the apical membrane increases the speed $\mathrm{CBF}$ (56); in a similar fashion, KCa3.1 inhibition might have a hyperpolarizing effect in the apical membrane (by decreasing $\mathrm{Na}^{+}$absorption) boosting $\mathrm{CBF}$. Our results also indicate that $\mathrm{KCa} 3.1$ inhibition had a greater effect on $\mathrm{CBF}$ than $\mathrm{ENaC}$ inhibition. It might be possible that the reduction of anion secretion also favored apical membrane hyperpolarization, a hypothesis that is supported by the more positive $\mathrm{V}_{\text {te }}$ recorded in the $\mathrm{Kcnn}^{-1}{ }^{-}$tissue that might be due to $\mathrm{Na}^{+}$accumulation in the mucosa and reduced anion secretion. Besides that, KCa3.1 inhibition has been widely proven by different groups to prevent or ameliorate several inflammatory events, including those occurring in the airways (57-59). Since our model is a systemic null animal, other cells might also participate in the reduction of inflammatory damage after Kcnn4 silencing. Such is the case of neutrophils, whose number is reduced in the $K c n n 4^{-/}$lungs affected by acute lung injury (41). Similar results are obtained in the $\mathrm{CF} C f t \mathrm{r}^{\mathrm{F} 508 / \Delta \mathrm{F} 508}$ mouse, which after Kcnn4 silencing, shows almost completely reduced lethality associated with intestinal obstructions, but this is not accompanied by improvements in intestinal anion secretion (42). Finally, the use of KCa3.1 inhibitors must be taken with caution because off-targets are reported (60-62), and in our case, the use of 2 different inhibitors (clotrimazol and TRAM-34) resulted in noncomparable results in Ussing chamber experiments. Senicapoc, a new and more potent inhibitor of $\mathrm{KCa} 3.1$, has been tested in clinical trials for sickle cell anemia and is shown to be safe and well tolerated in patients, offering a faster option for treatment of muco-obstructive diseases in humans (63). 
Although patients with CF have found therapies in molecules that help correct mutant CFTR malfunctions $(64,65)$, and new such molecules are being discovered (66), almost $10 \%$ of patients with CF are still left with no such treatment. The potential use of $\mathrm{KCa} 3.1$ inhibitors corresponds with what has been called a "mutation-agnostic" treatment that might be used specially in patients with CF that are not eligible for the new available therapies, and it also seems suitable for easing other muco-obstructive diseases affecting humans. The absence of severe phenotypes in the Kcnn4-null animal $(35,67-69)$ suggests the use of KCa3.1 inhibitors may be safe in human diseases.

\section{Methods}

Reagents. Unless stated otherwise, all reagents (amiloride, forskolin, IBMX and clotrimazole) were obtained from MilliporeSigma, with the exception of TRAM-34, which was obtained from TOCRIS.

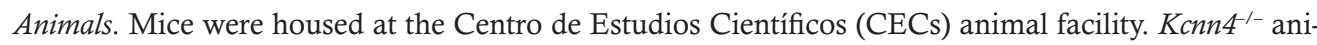
mal generation and their genotyping have been described previously (67). The Scnn $1 b^{\text {tg/+ }}$ mouse was backcrossed on a C57BL/6J background as previously described (70). Animals were maintained in the specific pathogen-free mouse facility of CECs with access to food and water ad libitum. Six-week-old male or female mice were used, all backcrossed for at least 20 generations on a C57BL/6J background.

Primary HBEC culture. The procedures for isolation and culture of HBECs were described in detail in a previous study (71). Briefly, HBECs were cultured in flasks in a serum-free medium (LHC9/RPMI 1640). After 4-5 passages, cells were seeded at high density $\left(500,000 / \mathrm{cm}^{2}\right)$ on Snapwell 3801 porous inserts (Corning Costar). Twenty-four hours after seeding, the medium was switched to DMEM/F12 (1:1) plus 2\% New Zealand fetal bovine serum (Thermo Fisher Scientific), hormones, and supplements. The medium was replaced daily on both sides of the permeable supports for up to 6-7 days (liquid-liquid culture). The apical medium was subsequently removed and the cells received nutrients only from the basolateral side (air-liquid culture $[\mathrm{ALC}]$ ). This condition favored further differentiation of the epithelium. Cells were maintained under ALC for 2 weeks before the experiments were performed. Experiments testing TRAM-34 used cells grown on BMIX media and experiments testing clotrimazol used CBMIX, as previously described (71). Only paired experiments from the same cells were included in the analysis.

Ussing chamber experiments. Tracheae were placed in $\mathrm{P} 2306 \mathrm{~B}$ of $0.057 \mathrm{~cm}^{2}$ tissue holders and placed in Ussing chambers (Physiologic Instruments Inc.). Tissues were bathed with bicarbonate-buffered solution ( $\mathrm{pH}$ 7.4) of the following composition (in $\mathrm{mM}$ ): $120 \mathrm{NaCl}, 25 \mathrm{NaHCO}_{3}, 3.3 \mathrm{KH}_{2} \mathrm{PO}_{4}, 0.8 \mathrm{~K}_{2} \mathrm{HPO}_{4}, 1.2 \mathrm{MgCl}_{2}, 1.2$ $\mathrm{CaCl}_{2}$, and $10 \mathrm{D}-\mathrm{Glucose}$, gassed with $5 \% \mathrm{CO}_{2}$ and $95 \% \mathrm{O}_{2}$, and kept at $37^{\circ} \mathrm{C}$. The transepithelial potential difference referred to the serosal side was measured using a VCC MC2 amplifier (Physiologic Instruments Inc.). The short-circuit currents $\left(\mathrm{I}_{\mathrm{SC}}\right)$ were calculated using Ohm's law, as previously described (72). Differences $\left(\Delta \mathrm{I}_{\mathrm{SC}}\right)$, were calculated from $\mathrm{I}_{\mathrm{SC}}$ after minus before the addition of drugs. The experiments were performed in the presence of $10 \mu \mathrm{M}$ amiloride in the apical side to block $\mathrm{Na}^{+}$absorptive currents. The $1 \mu \mathrm{M}$ forskolin plus $100 \mu \mathrm{M}$ 3-isobutyl-1-methylxanthine (IBMX) cocktail was used to stimulate cAMP-dependent anion secretion, and $100 \mu \mathrm{M}$ UTP to evoke $\mathrm{Ca}^{2+}$-activated anion secretion. For experiments on HBECs, Snapwell supports carrying differentiated bronchial epithelia were mounted in a vertical chamber resembling an Ussing system with internal fluid circulation. Both apical and basolateral hemichambers were filled with $5 \mathrm{~mL}$ of a Krebs bicarbonate solution containing (in mM): $126 \mathrm{NaCl}, 0.38 \mathrm{KH} 2 \mathrm{PO} 4,2.13 \mathrm{~K} 2 \mathrm{HPO} 4,1 \mathrm{MgSO} 4,1$ $\mathrm{CaCl} 2,24 \mathrm{NaHCO} 3$, and 10 glucose. Both sides were continuously bubbled with a gas mixture containing $5 \% \mathrm{CO}_{2}$ and $95 \%$ air and the temperature of the solution was kept at $37^{\circ} \mathrm{C}$. The transepithelial voltage was short-circuited with a voltage clamp (EVC-4000, World Precision Instruments) connected to the apical and basolateral chambers via $\mathrm{Ag} / \mathrm{AgCl}$ electrodes and agar bridges ( $1 \mathrm{M} \mathrm{KCl}$ in $1 \%$ agar). The offset between voltage electrodes and the fluid resistance were canceled before experiments. The short-circuit current was recorded with a PowerLab 4/25 (ADInstruments) analogical to digital converter connected to a computer.

$C B F$. Primary cultures of mouse airway epithelia were analyzed, as previously described (73). For all experiments, the central area on each ALI culture was imaged, and the digital image signal was routed from the camera directly into a digital image acquisition board (National Instruments) within a Dell XPS 710 workstation. Images were analyzed with virtual instrumentation software (Sissons-Ammons Video Analysis, National Instruments), which is highly customized to quantify CBF. All of the recordings in the present experiments were made at original magnification, $\times 630$. Whole-field analysis was performed, with each point measured representing one cilium. For each sample, the reported frequencies represent the arithmetic means of these values. Changes in CBF are normalized to the basal rate and reported as a ratio of stimulated/basal. 
Plastic beads clearance and in vivo MCC. Speed of polystyrene beads in trachea samples was determined with some modifications of the previously described method (74). Briefly, mice were deeply anesthetized via i.p. injection of $120 \mathrm{mg} / \mathrm{kg}$ ketamine and $16 \mathrm{mg} / \mathrm{kg}$ xylazine and exsanguinated, and the trachea was isolated and mounted using insect's needles in an extra thick blot paper (Bio-Rad), which was perfused with Ringer's solution (gassed with $95 \% / 5 \% \mathrm{O}_{2} / \mathrm{CO}_{2}$ ) at a rate of $1 \mathrm{ml} / \mathrm{min}$, at $37^{\circ} \mathrm{C}$, and maintained in a humidified chamber. Polystyrene black-dyed microspheres (diameter $6 \mu \mathrm{m}, 2.6 \%$ solid-latex, Polybead, Polyscience Inc.) were washed and diluted with physiological solution ( $0.5 \%$ latex) and $4 \mu \mathrm{l}$ of particle solution were added onto the bronchial edge of the trachea. Particle transport was visualized by images every 5 seconds ( $2-5$ minutes sampling) of at least 3 different fields using a Motic camera (Moticam 5.0). Stored images were measured to determine the velocity of single particles using NIH ImageJ software and MCC was expressed in $\mu \mathrm{m} / \mathrm{sec}$. Allergen clearance from the whole lung was evaluated by the elimination of fluorescently labeled OVA (Alexa Fluor 647) after intratracheal instillation, as previously described (13). $1 \mathrm{mg} / \mathrm{mL}$ OVA was labeled with Alexa Fluor 647 with the microscale Protein labeling kit (Alexa Fluor 647 Microscale Protein Labelling Kit, Life Technologies). Intratracheal instillation of $2.5 \mu \mathrm{g}$ OVA dissolved in $20 \mu \mathrm{PBS}$ was performed and held over 6 hours. To determine allergen clearance in the whole lung, mice were anesthetized via intraperitoneal injection with $10 \mathrm{mg} / \mathrm{kg}$ ketamine and $16 \mathrm{mg} / \mathrm{kg}$ xylazine. Lung was extracted after 6 hours. To determine the allergen clearance, lung was mixed with PBS and protease Inhibitor 1X (Roche). The lung was homogenized and fluorescence intensity was read in a microplate reader. Clearance was calculated using the following formula: $\% \mathrm{MCC}$ allergen $=[1$-fluorescence intensity at $6 \mathrm{hr}] /[$ fluorescence intensity at $0 \mathrm{hr}] \times 100$. The fluorescence intensity at baseline $(t=0)$ (arbitrary units) should not interfere between treatments.

Lung tissue isolation, $B A L F$, and histological analysis. Anesthetized mice were euthanized by exsanguination, and the chest cavity was opened to ligate the left main bronchus. A blunt needle (20 gauge) was inserted through a small incision in the upper trachea and tied in place with 3.0 silk. After ligation of the left main-stem bronchus, BAL was performed on the right lobes by instilling a volume of sterile PBS (137 mM $\mathrm{NaCl}, 2.7 \mathrm{mM} \mathrm{KCl}, 10 \mathrm{mM} \mathrm{Na} 2 \mathrm{HPO} 4,2 \mathrm{mM} \mathrm{KH} 2 \mathrm{PO} 4)$ at room temperature determined by the following formula: [mouse weight $(\mathrm{g}) \times 0.035 \mathrm{ml} / 2=\mathrm{mL}$ PBS instilled]

BAL was performed by gently injecting and retrieving the PBS volume 3 times. This procedure was carried out a second time with an equal volume of PBS and fractions were pooled. Return volume was consistently greater than $80 \%$ of the instilled volume. BAL cells were pelleted by centrifugation at $300 \mathrm{~g}$ for 5 minutes at $4^{\circ} \mathrm{C}$ and the cell-free supernatant (BALF) was collected and stored at $-80^{\circ} \mathrm{C}$ with protease inhibitors. BAL cells were resuspended in $50 \mu \mathrm{L}$ PBS; $10 \mu 1$ were counted with a hemocytometer (diluted in $10 \mu 1$ Trypan blue); and $40 \mu 1$ were diluted in $160 \mu 1$ and displaced in Cytospin slides (StatSpin Cytofuge 2), air dried, and stained with modified Giemsa for differential cell counts of at least 200 cells per slide. After BAL, the left and right lung were immersed in 10\% neutral-buffered formalin to prevent the dislodging of airway luminal contents. The right lung was used to evaluate the mean linear intercept and the left lung to quantify mucus density and attachment using NIH ImageJ software.

Lung histology and mucus morphometry. Morphometric analyses of airway mucus obstruction were performed in noninflated, immersion-fixed left lungs. Lungs were removed through a median sternotomy, fixed in $4 \%$ buffered formalin, and embedded in paraffin. Left lung was sectioned transversally at the level of the proximal intrapulmonary main axial airway near the hilus (proximal airways), and at the distal intrapulmonary axial airway, at $1500 \mu \mathrm{m}$ distal to the hilus (distal airways). To quantify secreted mucus, morphometric analysis of stained sections was carried out by determining mucus volume density using CellF software, as previously described (13). Briefly, the length of the basal membrane of the airway epithelium was measured by the interactive image measurement tool, and the Alcian blue-PAS staining-positive (AB-PAS-positive) surface area within this boundary was measured by phase analysis according to the automatic threshold settings of the software. The volume density of intraepithelial mucus, representing the volume of lumen mucus content per surface area of the mucus basal membrane $\left(\mathrm{nl} / \mathrm{mm}^{2}\right)$, was determined from the intraepithelial surface area of AB-PAS positive mucus and the length of the basal membrane of the airway epithelium. Luminal mucus content relative to the luminal area was determined in a similar fashion. Mucus attached to the epithelium was quantified with NIH ImageJ software. Using the interactive tool, polygon selections of the entire epithelial surface was surrounded to obtain the total perimeter. Then, epithelial surface in contact with the mucus was quantified and percentage determined. Formalin-fixed, paraffin-embedded lung sections cut at a thickness of $5 \mu \mathrm{m}$ were hydrated and pretreated with 3\% hydrogen peroxide in methanol followed by incubation for 1 hour with 1:5000 
Table 2. Primer sequences and annealing temperatures

\begin{tabular}{|c|c|c|c|}
\hline Scnn1b & NM_011325.2 & $\begin{array}{l}\text { Forward: GGCCCAGGCTACACCTACA } \\
\text { Reverse: AGCAGCGTAAGCAGGAAC }\end{array}$ & 58 \\
\hline Prostasin (Prss8) & NM_133351.3 & $\begin{array}{l}\text { Forward: TTGGAGGTACGCACAGATCC } \\
\text { Reverse: CCTCGGCTCTCCCTTAATCT }\end{array}$ & 58 \\
\hline
\end{tabular}

InVivoPlus anti-mouse Ly6G/Ly6C (Gr-1) (Bio X Cell). Further blocking with serum and developing of immune reaction was performed with VECTASTAIN ABC systems (Vector labs).

$m R N A$ isolation and $c D N A$ synthesis from epithelial cells. Mice were killed by cervical dislocation and tracheal tissues were immediately extracted; tracheae were incubated with Pronase $30 \mu \mathrm{M}$ at $37^{\circ} \mathrm{C}$ for 30 minutes. Trachea was placed in DMEM $10 \mathrm{mM}$ D-Glucose and epithelium was isolated by scraping with tweezers and further homogenized in $250 \mu \mathrm{L}$ Trizol (TRIzol Reagent) and RNA isolated following the manufacturer's instructions. The dried pellet of RNA was resuspended with $35 \mu \mathrm{L}$ nuclease free water and stored at $-80^{\circ} \mathrm{C}$. DNA contamination was avoided using DNAse treatment. The concentration and integrity of the RNA were determined by spectrophotometry. Total RNA was reverse transcribed into cDNA using the Superscript III RTPCR System according to the manufacturer's recommendations. cDNA synthesis was performed on $2 \mu \mathrm{g}$ RNA. cDNA integrity was checked using specific primers to cyclophilin and $50 \mathrm{ng}$ template cDNA was added to the reaction mixture. Cyclophilin (Ppia) amplification was performed starting with a 5 -minute template denaturation step at $95^{\circ} \mathrm{C}$, followed by 30 cycles of denaturation at $95^{\circ} \mathrm{C}$ for 30 seconds and combined primer annealing/extension at $55^{\circ} \mathrm{C}$. The relative brightness intensity of ethidium bromide-stained bands resolved on a $1.5 \%$ agarose gel was evaluated.

Real-time PCR. Quantification of Scnn1a, Scnn1b, Scnn1g, and Prss8 expression was performed using SYBR Green detection in a LightCycler PCR machine according to the manufacturer's instructions. We determined the PCR efficiency of each individual assay by serially measuring $100 \mathrm{ng}$ cDNA from a pool of epithelia in triplicate. Only CT values of less than 40 were used for calculation of the PCR efficiency. All PCRs displayed an efficiency between $96 \%$ and $100 \%$. Amplifications were performed starting with a 3-minute template denaturation step at $94^{\circ} \mathrm{C}$, followed by 45 cycles of denaturation at $94^{\circ} \mathrm{C}$ for 20 seconds and combined primer annealing/extension at the gene-specific primer temperature for 30 seconds. All samples were amplified in triplicate and the mean was obtained for further calculations. Relative fold changes in target gene expression were quantified by the previously reported $\Delta \Delta \mathrm{CT}$ method (75). Briefly, CT values were obtained for individual samples using the Rotor-Gene 6000 software 1.7 (Corbett Life Science), where the targets and reference (Cyclophilin) had the same cDNA concentration. $\triangle \mathrm{CT}$ was calculated by subtracting the $\mathrm{CT}$ (target - reference). Primers and annealing temperatures are provided in Table 2 .

Statistics. All statistical analyses were performed using SigmaPlot V12.1. Rank sum test for paired groups and 1-way ANOVA for multiple comparisons were used as indicated on each figure or table. A $P$ value of less than 0.05 was considered significant. Error bars represent mean \pm SEM, and single experiments are included in figures.

Study approval. All experimental procedures in animals were approved by the CECs Institutional Animal Care and Use Committee (1151142-2015) and followed the relevant guidelines and regulations. The CECs animal facility is AAALAC accredited. The collection of bronchial epithelial cells to investigate the mechanisms of transepithelial ion transport were specifically approved by the ethics committee of the Istituto Giannina Gaslini following the guidelines of the Italian Ministry of Health (updated registration number: ANTECER, 042-09/07/2018). Each patient provided informed consent using a form that was also approved by the ethics committee. 


\section{Author contributions}

CAF conceived the study. Mouse electrophysiology and data analysis were performed by ARP and CAF. HBEC electrophysiology and data analysis were performed by A. Gianotti, OZM, and LJVG, and CAF CBF experiments and data analysis were performed by ARP, LA, and MV. MCC in vivo and ex vivo was performed by GV and A. Guequén. Data analysis was performed by A. Guequén and CAF. BALF leukograms and analysis were performed by GV. Neutrophil IHC was performed by GV. Analysis was performed by CAF. Histological analysis was performed by GV and CAF. Experimental design was performed by GV. A. Guequén, A. Gianotti, MAM, and CAF wrote the paper with help from GV and MAM. All authors revised and approved the final version.

\section{Acknowledgments}

We express our gratitude to the CECs animal facility personnel for their work. CECs is funded by the Centers of Excellence Base Financing Program of Comisión Nacional de Investigación Científica y Tecnológica. This work was supported by Fondo Nacional de Desarrollo Científico y Tecnológico 1151142 (to CAF); by grants from the German Federal Ministry of Education and Research (82DZL004A1), the German Research Foundation (SFB-TR84TP B08), and the Einstein Foundation Berlin (EP-2017-393) (to MAM); and by Telethon Foundation grant TMLGCBX16TT (to LJVG).

Address correspondence to: Carlos A. Flores, Centro de Estudios Científicos (CECs), Valdivia, 511046, Chile. Email: cflores@cecs.cl.

1. Bustamante-Marin XM, Ostrowski LE. Cilia and mucociliary clearance. Cold Spring Harb Perspect Biol. $2017 ; 9(4)$ :a028241.

2. Mall MA. Role of cilia, mucus, and airway surface liquid in mucociliary dysfunction: lessons from mouse models. $J$ Aerosol Med Pulm Drug Deliv. 2008;21(1):13-24.

3. Fahy JV, Dickey BF. Airway mucus function and dysfunction. N Engl J Med. 2010;363(23):2233-2247.

4. Tilley AE, Walters MS, Shaykhiev R, Crystal RG. Cilia dysfunction in lung disease. Annu Rev Physiol. 2015;77:379-406.

5. Seibold MA, et al. A common MUC5B promoter polymorphism and pulmonary fibrosis. N Engl J Med. 2011;364(16):1503-1512.

6. Harun SN, Wainwright C, Klein K, Hennig S. A systematic review of studies examining the rate of lung function decline in patients with cystic fibrosis. Paediatr Respir Rev. 2016;20:55-66.

7. Pezzulo AA, et al. Reduced airway surface $\mathrm{pH}$ impairs bacterial killing in the porcine cystic fibrosis lung. Nature. 2012;487(7405):109-113.

8. Itani OA, et al. Human cystic fibrosis airway epithelia have reduced $\mathrm{Cl}$ - conductance but not increased Na+ conductance. Proc Natl Acad Sci USA. 2011;108(25):10260-10265.

9. Chen $\mathrm{JH}$, et al. Loss of anion transport without increased sodium absorption characterizes newborn porcine cystic fibrosis airway epithelia. Cell. 2010;143(6):911-923.

10. Donaldson SH, Boucher RC. Update on pathogenesis of cystic fibrosis lung disease. Curr Opin Pulm Med. 2003;9(6):486-491.

11. O'Donoghue DL, Dua V, Moss GW, Vergani P. Increased apical Na+ permeability in cystic fibrosis is supported by a quantitative model of epithelial ion transport. J Physiol (Lond). 2013;591(15):3681-3692.

12. Mall M, Grubb BR, Harkema JR, O’Neal WK, Boucher RC. Increased airway epithelial Na+ absorption produces cystic fibrosis-like lung disease in mice. Nat Med. 2004;10(5):487-493.

13. Mall MA, et al. Development of chronic bronchitis and emphysema in beta-epithelial Na+ channel-overexpressing mice. Am J Respir Crit Care Med. 2008;177(7):730-742.

14. Hirsh AJ, et al. Evaluation of second generation amiloride analogs as therapy for cystic fibrosis lung disease. J Pharmacol Exp Ther. 2004;311(3):929-938.

15. Hirsh AJ, et al. Pharmacological properties of N-(3,5-diamino-6-chloropyrazine-2-carbonyl)- N'-4-[4-(2,3-dihydroxypropoxy) phenyl]butyl-guanidine methanesulfonate (552-02), a novel epithelial sodium channel blocker with potential clinical efficacy for cystic fibrosis lung. J Pharmacol Exp Ther. 2008;325(1):77-88.

16. Hofmann T, Senier I, Bittner P, Hüls G, Schwandt HJ, Lindemann H. Aerosolized amiloride: dose effect on nasal bioelectric properties, pharmacokinetics, and effect on sputum expectoration in patients with cystic fibrosis. $J$ Aerosol Med. 1997;10(2):147-158.

17. O'Riordan TG, et al. Acute hyperkalemia associated with inhalation of a potent ENaC antagonist: Phase 1 trial of GS-9411. J Aerosol Med Pulm Drug Deliv. 2014;27(3):200-208.

18. Gianotti A, et al. Epithelial sodium channel silencing as a strategy to correct the airway surface fluid deficit in cystic fibrosis. Am J Respir Cell Mol Biol. 2013;49(3):445-452.

19. Tagalakis AD, et al. Effective silencing of ENaC by siRNA delivered with epithelial-targeted nanocomplexes in human cystic fibrosis cells and in mouse lung. Thorax. 2018;73(9):847-856.

20. Koefoed-Johnsen V, Ussing HH. The nature of the frog skin potential. Acta Physiol Scand. 1958;42(3-4):298-308.

21. Bardou O, Trinh NT, Brochiero E. Molecular diversity and function of $\mathrm{K}+$ channels in airway and alveolar epithelial cells. Am $J$ Physiol Lung Cell Mol Physiol. 2009;296(2):L145-L155.

22. Joo NS, Jeong JH, Cho HJ, Wine JJ. Marked increases in mucociliary clearance produced by synergistic secretory agonists or inhibition of the epithelial sodium channel. Sci Rep. 2016;6:36806.

23. Kerem E, et al. Pulmonary epithelial sodium-channel dysfunction and excess airway liquid in pseudohypoaldosteronism. $N$ Engl 
J Med. 1999;341(3):156-162.

24. Tildy BE, Rogers DF. Therapeutic options for hydrating airway mucus in cystic fibrosis. Pharmacology. 2015;95(3-4):117-132.

25. Turnheim K, Plass H, Wyskovsky W. Basolateral potassium channels of rabbit colon epithelium: role in sodium absorption and chloride secretion. Biochim Biophys Acta. 2002;1560(1-2):51-66.

26. Vallon V, et al. KCNQ1-dependent transport in renal and gastrointestinal epithelia. Proc Natl Acad Sci USA. 2005;102(49):17864-17869.

27. Hebert SC, Desir G, Giebisch G, Wang W. Molecular diversity and regulation of renal potassium channels. Physiol Rev. 2005;85(1):319-371.

28. Preston $\mathrm{P}$, et al. Disruption of the $\mathrm{K}+$ channel beta-subunit $\mathrm{KCNE} 3$ reveals an important role in intestinal and tracheal $\mathrm{Cl}$ transport. J Biol Chem. 2010;285(10):7165-7175.

29. Thompson-Vest N, Shimizu Y, Hunne B, Furness JB. The distribution of intermediate-conductance, calcium-activated, potassium (IK) channels in epithelial cells. J Anat. 2006;208(2):219-229.

30. Villanueva S, et al. Cleft palate, moderate lung developmental retardation and early postnatal lethality in mice deficient in the Kir7.1 inwardly rectifying K+ channel. PLOS ONE. 2015;10(9):e0139284.

31. Mall M, et al. Modulation of Ca2+-activated Cl- secretion by basolateral $\mathrm{K}+$ channels in human normal and cystic fibrosis air way epithelia. Pediatr Res. 2003;53(4):608-618.

32. Mall M, et al. Role of K(V)LQT1 in cyclic adenosine monophosphate-mediated Cl(-) secretion in human airway epithelia. Am $J$ Respir Cell Mol Biol. 2000;23(3):283-289.

33. Devor DC, Bridges RJ, Pilewski JM. Pharmacological modulation of ion transport across wild-type and DeltaF508 CFTR-expressing human bronchial epithelia. Am J Physiol, Cell Physiol. 2000;279(2):C461-C479.

34. Yin $\mathrm{W}$, et al. The potassium channel KCNJ13 is essential for smooth muscle cytoskeletal organization during mouse tracheal tubulogenesis. Nat Commun. 2018;9(1):2815.

35. Flores CA, Melvin JE, Figueroa CD, Sepúlveda FV. Abolition of Ca2+-mediated intestinal anion secretion and increased stool dehydration in mice lacking the intermediate conductance Ca2+-dependent K+ channel Kcnn4. J Physiol (Lond). 2007;583(Pt 2):705-717.

36. Julio-Kalajzić $\mathrm{F}$, et al. $\mathrm{K}_{2 \mathrm{P}}$ TASK-2 and KCNQ1-KCNE3 $\mathrm{K}^{+}$channels are major players contributing to intestinal anion and fluid secretion. J Physiol (Lond). 2018;596(3):393-407.

37. Hummler E, Vallon V. Lessons from mouse mutants of epithelial sodium channel and its regulatory proteins. $J$ Am Soc Nephrol. 2005;16(11):3160-3166.

38. Hummler E, et al. Early death due to defective neonatal lung liquid clearance in alpha-ENaC-deficient mice. Nat Genet. 1996;12(3):325-328

39. Barker PM, et al. Role of gammaENaC subunit in lung liquid clearance and electrolyte balance in newborn mice. Insights into perinatal adaptation and pseudohypoaldosteronism. J Clin Invest. 1998;102(8):1634-1640.

40. McDonald FJ, et al. Disruption of the beta subunit of the epithelial Na+ channel in mice: hyperkalemia and neonatal death associated with a pseudohypoaldosteronism phenotype. Proc Natl Acad Sci USA. 1999;96(4):1727-1731.

41. Henríquez C, et al. The calcium-activated potassium channel $\mathrm{KCa} 3.1$ plays a central role in the chemotactic response of mam malian neutrophils. Acta Physiol (Oxf). 2016;216(1):132-145

42. Philp AR, et al. Kcnn4 is a modifier gene of intestinal cystic fibrosis preventing lethality in the Cftr-F508del mouse. Sci Rep. 2018;8(1):9320.

43. Di L, et al. Inhibition of the K+ channel KCa3.1 ameliorates T cell-mediated colitis. Proc Natl Acad Sci USA. 2010;107(4):1541-1546.

44. Ehre C, et al. Overexpressing mouse model demonstrates the protective role of Muc5ac in the lungs. Proc Natl Acad Sci USA. 2012;109(41):16528-16533.

45. Raclawska DS, et al. Mucins and their sugars. Critical mediators of hyperreactivity and inflammation. Ann Am Thorac Soc. 2016;13 Suppl 1:S98-S99.

46. Livraghi-Butrico A, et al. Contribution of mucus concentration and secreted mucins Muc5ac and Muc5b to the pathogenesis of muco-obstructive lung disease. Mucosal Immunol. 2017;10(2):395-407.

47. Joiner WJ, Wang LY, Tang MD, Kaczmarek LK. hSK4, a member of a novel subfamily of calcium-activated potassium channels. Proc Natl Acad Sci USA. 1997;94(20):11013-11018

48. Vandorpe DH, et al. cDNA cloning and functional characterization of the mouse Ca2+-gated K+ channel, mIK1. Roles in regulatory volume decrease and erythroid differentiation. J Biol Chem. 1998;273(34):21542-21553.

49. Lee RJ, Foskett JK. Ca ${ }^{2+}$ signaling and fluid secretion by secretory cells of the airway epithelium. Cell Calcium. 2014;55(6):325-336

50. Welsh MJ, McCann JD. Intracellular calcium regulates basolateral potassium channels in a chloride-secreting epithelium. Proc Natl Acad Sci USA. 1985;82(24):8823-8826

51. De Proost I, et al. Functional live cell imaging of the pulmonary neuroepithelial body microenvironment. Am J Respir Cell Mol Biol. 2008;39(2):180-189.

52. Zhang L, Sanderson MJ. Oscillations in ciliary beat frequency and intracellular calcium concentration in rabbit tracheal epithelial cells induced by ATP. J Physiol (Lond). 2003;546(Pt 3):733-749.

53. Anderson WH, et al. The relationship of mucus concentration (hydration) to mucus osmotic pressure and transport in chronic bronchitis. Am J Respir Crit Care Med. 2015;192(2):182-190.

54. Li Y, Wang W, Parker W, Clancy JP. Adenosine regulation of cystic fibrosis transmembrane conductance regulator through prostenoids in airway epithelia. Am J Respir Cell Mol Biol. 2006;34(5):600-608.

55. Donaldson SH, Lazarowski ER, Picher M, Knowles MR, Stutts MJ, Boucher RC. Basal nucleotide levels, release, and metabolism in normal and cystic fibrosis airways. Mol Med. 2000;6(11):969-982.

56. Ohba T, et al. Enhancement of $\mathrm{Ca}(2+)$ influx and ciliary beating by membrane hyperpolarization due to ATP-sensitive $\mathrm{K}(+)$ channel opening in mouse airway epithelial cells. J Pharmacol Exp Ther. 2013;347(1):145-153.

57. Girodet PO, et al. $\mathrm{Ca}(2+)$-activated $\mathrm{K}(+)$ channel-3.1 blocker TRAM-34 attenuates airway remodeling and eosinophilia in a murine asthma model. Am J Respir Cell Mol Biol. 2013;48(2):212-219.

58. Lin H, Zheng C, Li J, Yang C, Hu L. Lentiviral shRNA against KCa3.1 inhibits allergic response in allergic rhinitis and sup presses mast cell activity via PI3K/AKT signaling pathway. Sci Rep. 2015;5:13127. 
59. Yu Z, Wang Y, Qin L, Chen H. Functional cooperation between KCa3.1 and TRPV4 channels in bronchial smooth muscle cell proliferation associated with chronic asthma. Front Pharmacol. 2017;8:559.

60. Agarwal JJ, Zhu Y, Zhang QY, Mongin AA, Hough LB. TRAM-34, a putatively selective blocker of intermediate-conductance, calcium-activated potassium channels, inhibits cytochrome P450 activity. PLoS ONE. 2013;8(5):e63028.

61. Bonito B, Sauter DR, Schwab A, Djamgoz MB, Novak I. $\mathrm{K}_{\mathrm{Ca}} 3.1$ (IK) modulates pancreatic cancer cell migration, invasion and proliferation: anomalous effects on TRAM-34. Pflugers Arch. 2016;468(11-12):1865-1875.

62. Schilling T, Eder C. TRAM-34 inhibits nonselective cation channels. Pflugers Arch. 2007;454(4):559-563.

63. Ataga KI, et al. Efficacy and safety of the Gardos channel blocker, senicapoc (ICA-17043), in patients with sickle cell anemia. Blood. 2008;111(8):3991-3997.

64. Heijerman HGM, et al. Efficacy and safety of the elexacaftor plus tezacaftor plus ivacaftor combination regimen in people with cystic fibrosis homozygous for the F508del mutation: a double-blind, randomised, phase 3 trial. Lancet. 2019;394(10212):1940-1948.

65. Middleton PG, et al. Elexacaftor-tezacaftor-ivacaftor for cystic fibrosis with a single Phe508del Allele. N Engl J Med. 2019;381(19):1809-1819.

66. Pedemonte N, et al. Discovery of a picomolar potency pharmacological corrector of the mutant CFTR chloride channel. Sci Adv. 2020;6(8):eaay9669.

67. Begenisich T, et al. Physiological roles of the intermediate conductance, Ca2+-activated potassium channel Kcnn4. J Biol Chem. 2004;279(46):47681-47687.

68. Grgic I, et al. Disruption of the Gardos channel (KCa3.1) in mice causes subtle erythrocyte macrocytosis and progressive splenomegaly. Pflugers Arch. 2009;458(2):291-302.

69. Si H, et al. Impaired endothelium-derived hyperpolarizing factor-mediated dilations and increased blood pressure in mice deficient of the intermediate-conductance Ca2+-activated K+ channel. Circ Res. 2006;99(5):537-544

70. Johannesson B, Hirtz S, Schatterny J, Schultz C, Mall MA. CFTR regulates early pathogenesis of chronic obstructive lung disease in $\beta E N a C$-overexpressing mice. PLoS One. 2012;7(8):e44059.

71. Musante I, et al. Peripheral localization of the epithelial sodium channel in the apical membrane of bronchial epithelial cells. Exp Physiol. 2019;104(6):866-875.

72. Gianotti A, et al. Pharmacological analysis of epithelial chloride secretion mechanisms in adult murine airways. Eur J Pharmacol. 2016;781:100-108.

73. Zhao KQ, et al. Molecular modulation of airway epithelial ciliary response to sneezing. FASEB J. 2012;26(8):3178-3187.

74. Ousingsawat J, Martins JR, Schreiber R, Rock JR, Harfe BD, Kunzelmann K. Loss of TMEM16A causes a defect in epithelial Ca2+-dependent chloride transport. J Biol Chem. 2009;284(42):28698-28703.

75. Livak KJ, Schmittgen TD. Analysis of relative gene expression data using real-time quantitative PCR and the 2(-Delta Delta C(T)) Method. Methods. 2001;25(4):402-408 